\title{
La MELASSE de CANNe a SUCre COMME BASE DE L'ALIMENTATION POUR L'ENGRAISSEMENT DES BOVINS
}

\author{
R. SANSOUCY *
}

\section{RESUME}

Utilisće dcpuis fort longtemps à un taux txès faible (moins de 15 p. 100), la mélasse n'est utilisée comme base de la ration que depuis les récents travaux de T.R. PRESTON à Cuba.

Cumpte tenu des caractéristiques particulières de la mélasse, son utilisation intensive exige gue

- la mélasse soit distribuée non diluée ad libitum », la consommation est alors de $2,5 \grave{a} 3 \mathrm{~kg}$ par $100 \mathrm{~kg}$ de poids vif

- ie fourage soit restreint au minimum (1,5 à 2 p. 100 du poids vif). Le ıpe de fourrage importe peu, cependant Letcaena leucocephala a donné à l’x̂le Maurice des résultats très intéressants,

- la complémentation azotée soit adaptée. L'urée peut apporter près de 60 p. 100 de l'azote total mais une source de protéine vraie peu soluble est indispensable.

Un apport éventuel d'un complément de céréales (maïs) améliore les perfor. mances mais est coûteux.

Te système pewt être actuellement considéré comme opérationnel et peut intéresser tous les pays producteurs de sucre de canne.

\section{SUMMARY}

Sugar cane molasses as basis of diet for fattening cattle

Molasses have been used for a long time, but at a very low rate (less than 15 p. 100). They have been used as basis of diet only after the recent works of T.-R. PRESTON in Cuba.

Due to their particular characteristics the intensive use of molasses needs the following conditions :

- molasses must be given "ad libitum " and non diluted. The intake is then between 2,5 and $3,0 \mathrm{~kg}$ per $100 \mathrm{~kg}$ live weight,

- forage must be restricted to the minimum : 1,5 to $2,0 \mathrm{~kg}$ per $100 \mathrm{~kg}$ live weight. Type of forage does not matter, howcver Leucaena laucoccphala has given very intersting results in Mauritius,

- the nitrogen complementation must be adapted. Urea can supply about $60 \mathrm{p}$. 100 of the total nitrogen but a source of true protein of low solubility is essential.

A possible complement of cereals (maïze) improves the performances but is expensive.

The system may be considered now as operational and may interest all the cane sugar producing countries.

\section{INTRODUCTION}

La mélasse de canne à suçe a été utilisée depuis fort longtemps. Cependant, elle a toujours été employée à des taux relativement faibles (R. SANSOUCY, 1973).

Les différentes formes d'utilisation traditionnelles sont :

- l'incorporation comme liant dans les aliments concentrés

- l'addition à certains ensilages pour faciliter la conservation

- l'arrosage d'aliments pet appétents pour faciliter la consommation,

(*) Spécialiste en production animale, F.A.O.
- Ie mélange avec des sources d'azote non protéiques (urée) et des minéraux pour constituer des suppléments liquides, en particulier aux fourrages pauvres.

Dans la plupart des travaux effectués, on signale des effets diarrhéiques, même lorsque la mélasse est utilisée à muins de 15 p. 100 dans la ration (Commonwealth Agricultural Bureaux, 1968). Les inconvénients signalés se rapportent généralement au taux élevé en matières minérales, en particulier en potassium (PICCIONNI, 1965). D'autre part, MORRISON (1967) lui même indique que de fortes quantités de mélasse peuvent entraîner une diminution de la valeur alimentaire de ce produit de l'ordre de 50 p. 100.

En fait, la mélasse de canne à sucre n'a vu son 
intérêt s'accroître que depuis les travaux effectués à Cuba par le docteur PRESTON et son équipe à l'Instituto de Ciencia Animal, qui en ont fait un aliment de base pour les bovins à l'engraissement (PRESTON et WILLIS, 1969).

\section{Composition chimique de la mélasse}

Avant d'étudier les modalités pratiques de l'utilisation intensive de la mélasse, il est utilc de considérer sa composition chimique :

1

Composition chimique de la mélasse.

\begin{tabular}{|c|c|c|c|c|}
\hline \multirow{3}{*}{ MATIERE SECHE } & MAURTCE $[a]$ & CUEA (b) & U.S.A. [c] & RHODESIE [d] \\
\hline & $\mathrm{p} .100$ & P.100 & 2.700 & 2.100 \\
\hline & 80,90 & 76,90 & 74,50 & 80,00 \\
\hline \multirow{2}{*}{$\begin{array}{l}\text { Saceharose } \\
\text { Sugreg réducteurs }\end{array}$} & 33,60 & $45,50\}$ & 52,20 & 29,60 \\
\hline & 13,50 & $22,10\}$ & & 17,00 \\
\hline Proteines brutec & 5,06 & 4,42 & 4,30 & 4,00 \\
\hline MAIIERES MINERALES & 10,00 & 7,20 & 8,10 & 7.80 \\
\hline Potassium & 3,42 & 2,60 & 2,38 & 3,54 \\
\hline Calcitm & 1,11 & 0,92 & 8,89 & 0,66 \\
\hline Phosphore & 0,10 & 0,08 & Q. ०8 & 0,07 \\
\hline Magné́sium & 0,60 & 0,59 & 0,35 & 0,34 \\
\hline Sodium & 1,11 & 1,08 & 0,17 & 0,13 \\
\hline $\begin{array}{l}\text { a) M.S.I.R. } \\
\text { b) Preston } \\
\text { c) N.R.E. } \\
\text { d) Fincham }\end{array}$ & $\begin{array}{l}- \text { ๆ96? } \\
\text { W:1115 = } 1969 \\
956 \\
966\end{array}$ & & & \\
\hline
\end{tabular}

Il s'en dégage quatre caractéristiques principales:

1) Sa richesse en matières sèches $(80$ p. 100 environ) bien que ce soit un liquide!

2) Sa richesse en énergie : elle contient environ 50 p. 100 de sucres totaux et a une valeur énergétique de $0,75 \mathrm{UF} / \mathrm{kg}$;

3) Sa pauvreté en matières protéiques brutes (4 à 5 p. 100 seulement) ;

4) Son taux élevé en matières minérales, en particulier pour la moélasse de l'île Maurice.

Il y a lieu de tenir compte de ces caractéristiques lorsqu'on utilise la mélasse de façon intensive dans l'alimentation des animaux.

\section{Consommation de mélasse}

Compte tenu des remarques faites en introduction, l'un des premiers problèmes à résoudre était donc de pouvoir faire consommer des quantités suffisamment importantes de mélasse.

Pour cela, la technique a consisté à réduire au minimum la quantité de fourrage mise à la disposition des animaux.

2

Comparaison du fourrage à volonté ou restreint. (Martin, Preston et Willis * 1968.)

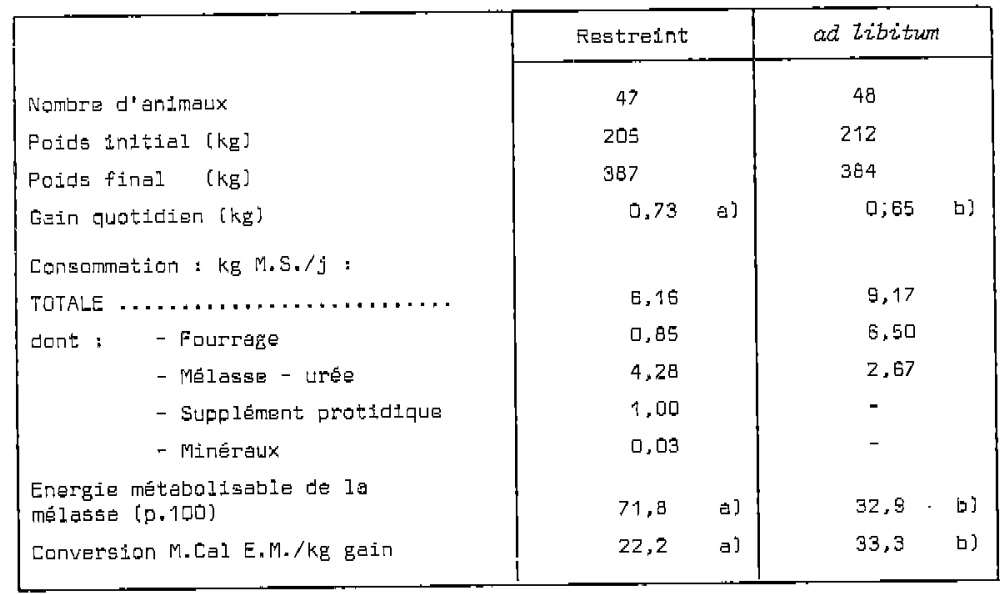

a), bl : Les chiffres n'syant aucune lettre en commun diffèrent: a $P$ 0,05

La réduction du fourrage vert (1,5 p. $100 \mathrm{du}$ poids vif) se traduit par :

1) Une augmentation de la consommation de mélasse qui apporte alors les $3 / 4$ de l'énergie métabolisable de la ration;

2) Une amélioration des performances;
3) Un taux de conversion mettement meilleur.

La consommation de mélasse, lorsqu'elle est distribuée à volonté avec un apport de fourrage restreint, est en moyenne d'environ $2,5 \mathrm{~kg}$ par $100 \mathrm{~kg}$ de poids vif, elle peut atteindre $3 \mathrm{~kg}$ sans que l'animal ne présente aucun signe d'intoxication. 


\section{L'apport de fourrage}

- Ouanitité : Elle peut être réduite au minimum nécessaure pour assurer un fonctionnement correct du rumen, soit :

$-1,5$ p. 100 du poids vif en fourrage frais, ou

$-0,3$ p. $100 \mathrm{du}$ poids vif en matière sèche.

On recommande de faire consommer au moins $1 \mathrm{~kg}$ de matière sèche de fourrage pour éviter les risques d'intoxication. Il faut s'assurer que chaque animal consomme bien du fourrage : l'observation de l'éleveur est importante.

\section{- Qualité :}

- Le type de fourrage vert peut varier : Pennisetum purpureum, Digitaria decumbens, Setaria sphacelata, etc.

- Nous avons également essayé de supprimer totalement le fourrage vert, en le remplaçant par de la bagasse grossière (en ce cas, il est nécessaire d'apporter un complément de vitamines).

3

Utilisation de la bagasse fraîche comme source de fourrage. (Sansoucy, Nielsen, Delattre et Preston - 1973.)

\begin{tabular}{|c|c|c|c|}
\hline \multirow[b]{2}{*}{ Nombre d'enimaux } & $\begin{array}{l}\text { Herbe } \\
3 \text { p.100 }\end{array}$ & $\begin{aligned} \text { Herbe } \\
1 \text { p.100 } \\
+\quad \text { Bagassa }\end{aligned}$ & $\begin{array}{c}\text { Bagasse } \\
\text { dd libitum }\end{array}$ \\
\hline & 8 & 8 & $6 \quad[1]$ \\
\hline Poids vif : = Initial (kg) & 231,9 & 233,9 & 222,7 \\
\hline$=$ final (kg) & 324,0 & 323,3 & 286,2 \\
\hline Gelin moyen quotidien [kg] & 0.532 & 0.523 & 0,365 \\
\hline Durée [ jours] & 173 & 171 & 174 \\
\hline Consommations : kg/an./ $\mathrm{d}$ : & & & \\
\hline - Bagasse & - & 0,62 & 0.75 \\
\hline - Fourrage vert & 7.83 & 2,75 & - \\
\hline - Mélassa & 6,21 & $\mathrm{E}, 4 \mathrm{~B}$ & 5.84 \\
\hline - Џrée & 0,124 & 0.130 & 0.117 \\
\hline- Se1 & 巾. 031 & 0,032 & 0.30 \\
\hline - Fartne de poisson & 0,281 & 0.299 & $\square, 263$ \\
\hline - Minéraux & 0,039 & 0,038 & 0.040 \\
\hline 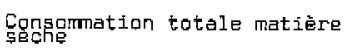 & 7,12 & 6,71 & 5.55 \\
\hline $\begin{array}{l}\text { Consommation de melasse } \\
\text { kg }{ }^{\prime} 100 \text { kg de Poids vifj }\end{array}$ & 2,23 & 2,33 & 2,30 \\
\hline $\begin{array}{l}\text { Consommiation tatale de matiè } \\
\text { seche }[\mathrm{kg} / 10 \mathrm{~kg} \text { de Polds v1 }\end{array}$ & 2,56 & 2,41 & 2,18 \\
\hline
\end{tabular}

(1) 2 animaux intoxiqués ont éte êliminés

Le gain de poids a ćté inférieur avec la bagasse seule. En fait, il semble bien que le problème pour ce groupe a été le manque d'appétence de la bagasse grossière, qui s'est traduit par une consommation très faible de bagasse : $0,76 \mathrm{~kg} / \mathrm{j}$, soit $0,4 \mathrm{~kg}$ seulement de matière sèche, quantité bien inférieure au minimum recommandé $(1 \mathrm{~kg} / \mathrm{j})$. Lorsqu'en fin d'expérience, nous avons mélangé la bagasse à la mélasse, les animaux de ce groupe en ont mangé $2,5 \mathrm{~kg} / \mathrm{j}$ (soit
$1,25 \mathrm{~kg}$ de matière brute) et leur gain a atteint $500 \mathrm{~g} / \mathrm{j}$

- Un type de fourrage qui s'est avéré très intéressant, c'est le Leucaena leucocephala. Nous citerons comme exemple le cas d'un éleveur ayant obtenu des résultats extrêmement faibles avcc du Pennisetum purpureum distribué à l'auge à raison de 3 p. 100 du poids vif et qui ont été améliorés de façon surprenante par apport de Leucaena leucocephala en pâturage restreint ( 2 h 30 par jour). 
CROISSANCE DE TAURILLONS ZEBUS NOURRIS A BASE DE MELASSE AVEC HERBE A ELEPHANT OU LEUCAENA COMME SOURCE DE FOURRAGE (SANSOUCY 1973) *

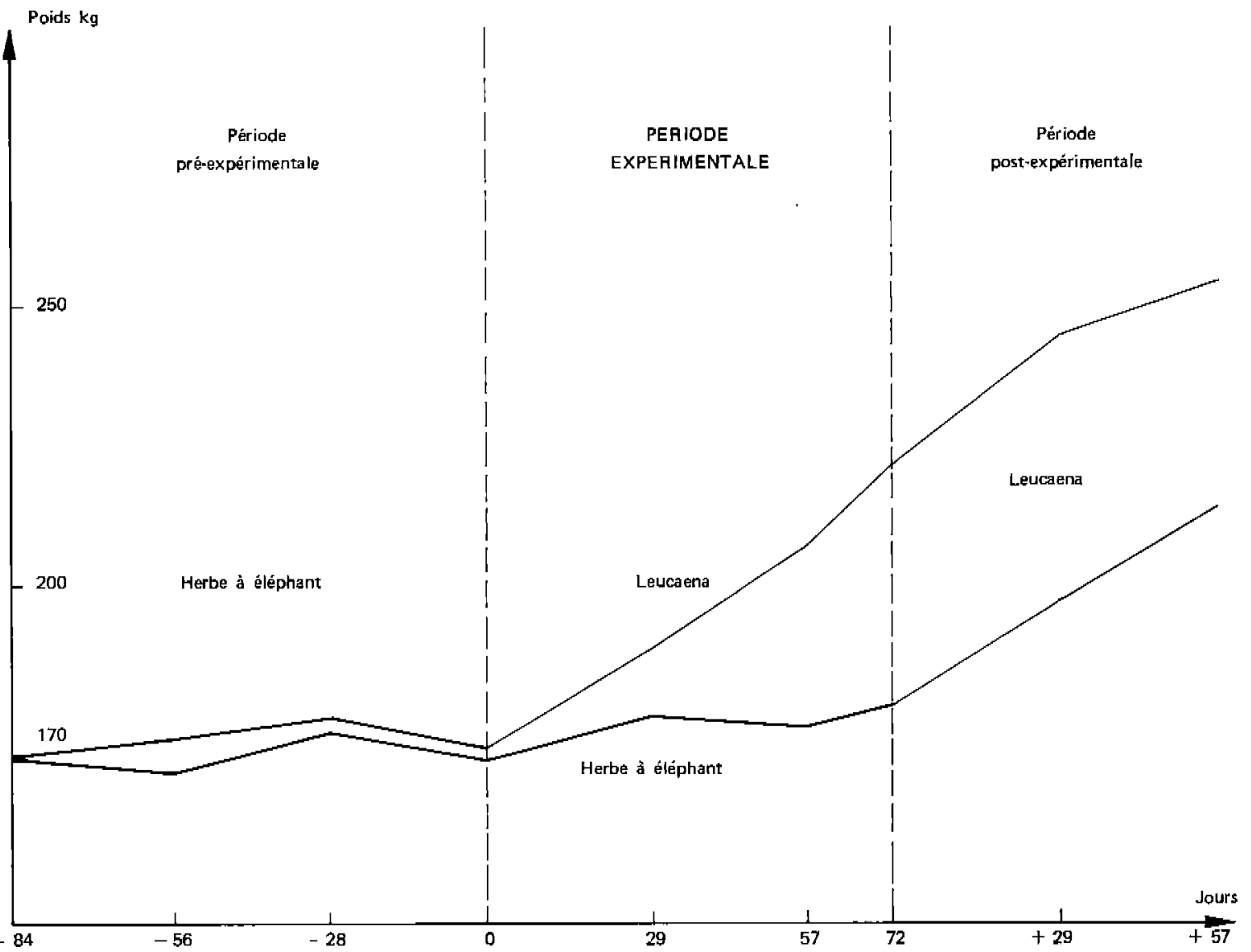

S'il est difficile d'expliquer d'aussi mauvais résultats avec le Pennisetum, il est par contre intéressant de remarquer le redressement spectaculaire obtenu avec Leucaena.

\section{Influence de la concentration de la mélasse}

S'il était acquis que le fourrage devait etre distribué en quantité restreinte, il a semblé que les risques seraient moins grands en utilisant une mélasse diluée. A cet effet, différentes concentrations ont été utilisées sur des animaux changés brusquement du pâturage à un régime à base de mélasse :

4

Influence de la concentration de la mélasse. (Preston, Elias et Willis - 1968.)

\begin{tabular}{|c|c|c|c|c|}
\hline & Concentr & de la & ח & Brix \\
\hline & $15^{\circ}$ & $35^{\circ}$ & $55^{\circ}$ & $75^{\circ}$ \\
\hline Galn quotidien (kg) & 0,70 & 0,77 & $\square, 77$ & 0,79 \\
\hline Eonsommatian (kg M.S./j) & 6,32 & 6,52 & 6,71 & 8,38 \\
\hline $\begin{array}{l}\text { Energie mútab. en mélasse } \\
\text { (p.100) }\end{array}$ & 73,7 & 76.7 & 76,9 & 75,7 \\
\hline $\begin{array}{l}\text { Conversion } \\
\text { (M.Cal. d'E.M./kg galn] }\end{array}$ & 25,1 & 25,1 & 24,0 & 22,1 \\
\hline
\end{tabular}


Trois animaux moururent de toxicité dans les deux groupes ayant reçu la mélasse la plus concentrée. Par ailleurs, les performances ont été meilleures pour ces deux groupes. D'autre part, l'addition d'eau présente l'inconvénient majeur de favoriser la fermentation alcoolique de la mélasse. Actuellement, on limite la consommation de mélasșe pendant la période de transition par un apport de fourrage à volonté que l'on réduit progressivement, mais la mélasse est utilisée telle quelle avec le strict minimum d'eau nécessaire pour dissoudre l'urée et le sel.

\section{La complémentation azotée}

Du fait de sa pauvreté en matières protéiques, la mélasse nécessite une complémentation azotée importante.

Les ruminanis pouvant utiliser l'urée comme source d'azote, celle-ci est naturellement utilisée, d'autant plus qu'elle peut être facil-znent mélangée de facon très homogène à la melasse et que selle-ci, riche en sucres solubles, se prête particulièrement bien à l'utilisation de l'uxéée par les micro-organismes du rumen. C'est ainsi que l'apport d'azote par l'urée, incorporée à raison de 2 à 2,5 p. 100 à la mélasse, peut représenter 60 p. 100 de l'apport total d'azote de la ration.

Cependant, il est nécessaire d'apporter également un complément de protéines vraies. La nature de ce complément a son importance. Pour completer efficacement les protéines d'origine microbienne produites par les micro-organismes vivant dans le rumen, les protéines supplémentaires ne doivent pas être elles-même dégradées au niveau du rumen, elles doivent donc être faiblement solubles (PRESTON, 1972). La farine de poisson apparaît comme la meilleure source. Comparée à du tourteau de colza par exemple (beaucoup plus soluble), elle a donné de bien meilleurs résultats.

Effet de la source de protéine.

Comparaison de la farine de poisson avec le tourteau de colza pour l'engraissement de taurillons à base de mélasse-urée. (Preston et Molina - 1972.)

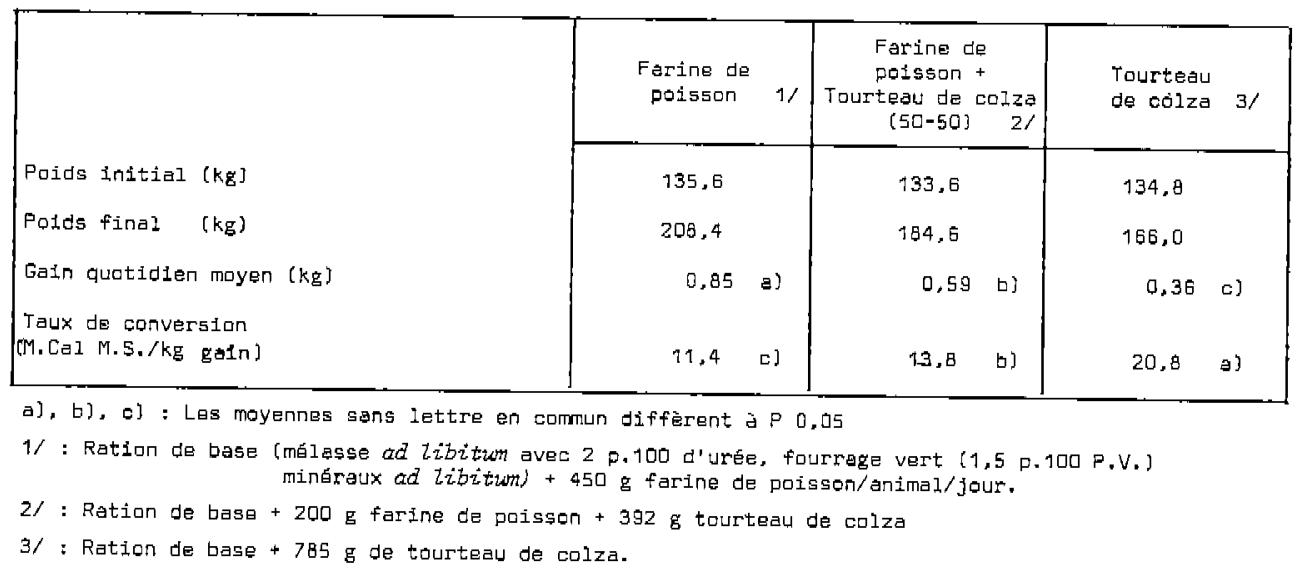

Toutefois, REDFERN (1972) au Kénya a obtenu d'excellentes performances en utilisant du tourteau de coton (également soluble) sans urée; nous avons également obtenu des résultats comparables avec du tourteau d'arachide sans urée (SANSOUCY et NIELSEN, 1972*), mais dans ce cas, la quantité de tour- teau nécessaire est telle que ceci risque de ne pas être économique.

Aussi, nous avons essayé de traiter le tourteau d'arachide au formaldéhyde pour réduire sa solubilité en l'utilisant avec de l'urée. Les résultats furent comparables avec la farine de poisson. 
GRAPHIQUE $\mathbf{N}^{\circ} 2$

COMPARAISON DE LA CROISSANCE DE TAURILLONS CREOLES NOURRIS

A BASE DE MELASSE LIQUIDE + 2 p. 100 UREE, AD LIBITUM AVEC :

$$
\begin{aligned}
& \text { A - } 300 \mathrm{~g} \text { farine poisson } \\
& \text { B - } 375 \mathrm{~g} \text { tourteau d'arachide } \\
& \mathrm{C}-\mathbf{3 7 5} \mathrm{g} \text { tourteau d'arachide traité } \\
& \text { au formal de hyde }
\end{aligned}
$$

et paturage restreint (acacia $3 \mathrm{~kg} / \mathrm{j}$ )

(SANSOUCY et NIELSEN - 1973)

Poids, $\mathrm{kg}$

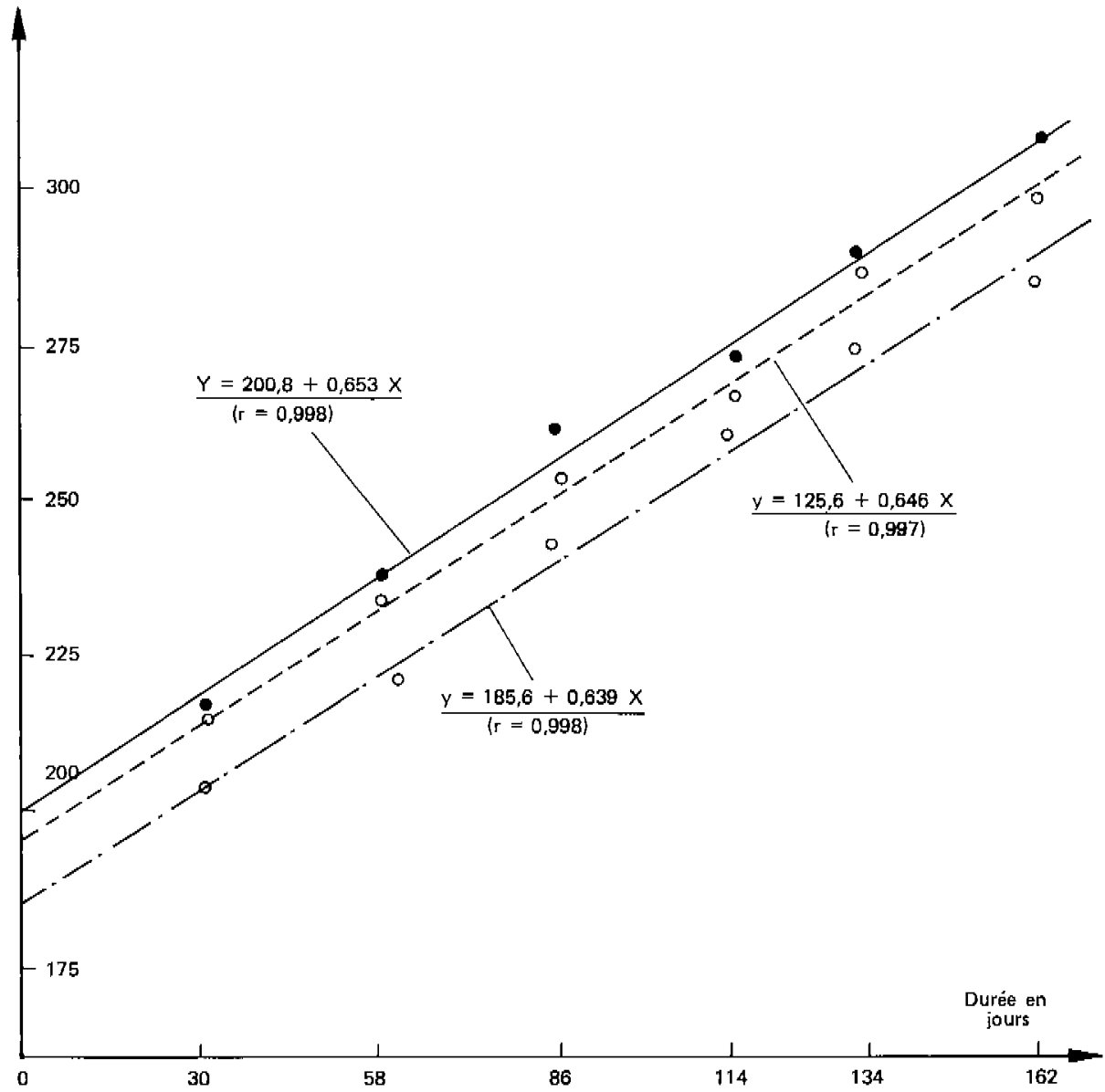


Malheureusement, dans ce cas, nous avions un facteur qui a pu introduire la confusion avec la présence de pâturage restreint de Leucaena. Il faudrait reprendre l'expérience avec une graminée comme source de fourrage, Leucaena étant riche en protéines.

Mais Leucaena a donné des résultats qui ont paru si intéressants qu'un essai a été prévu utilisant du
Leucaena comme source de fourrage, sans autre source de protéines vraies.

\section{Apport éventuel de céréales}

Le niveau des performances nous paraissant relativement faible à l'île Mauriçe, nous avons essayé de les renforcer par un apport de maiss-grain.

CROISSANCE EN FONCTION DU NIVEAU DE MAIS DANS LA RATION

SANSOUCY, NIELSEN, DELAITRE et PRESTON - 1973)

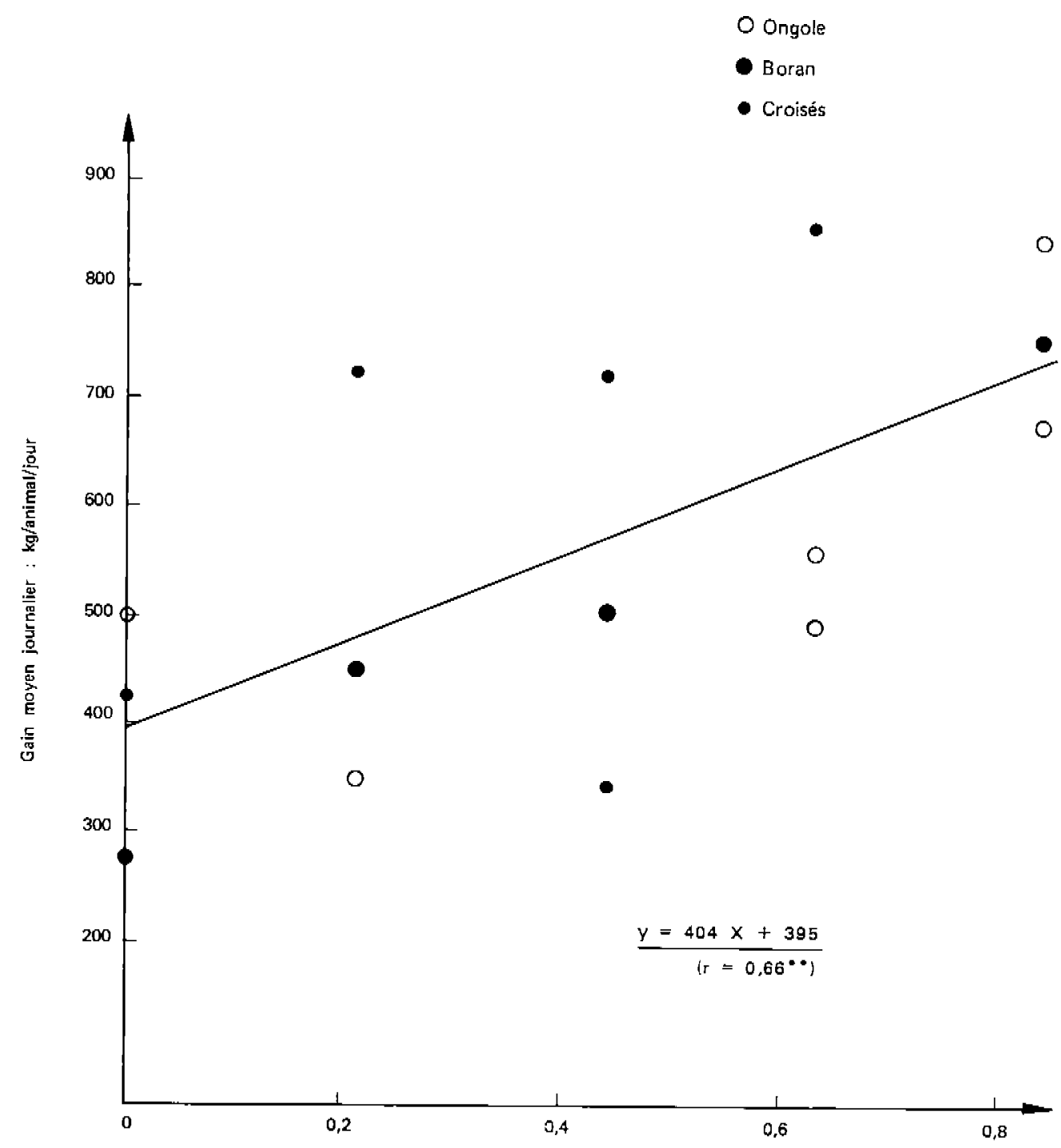

Niveau du mais dans la ration : p. 100 du poids vif 


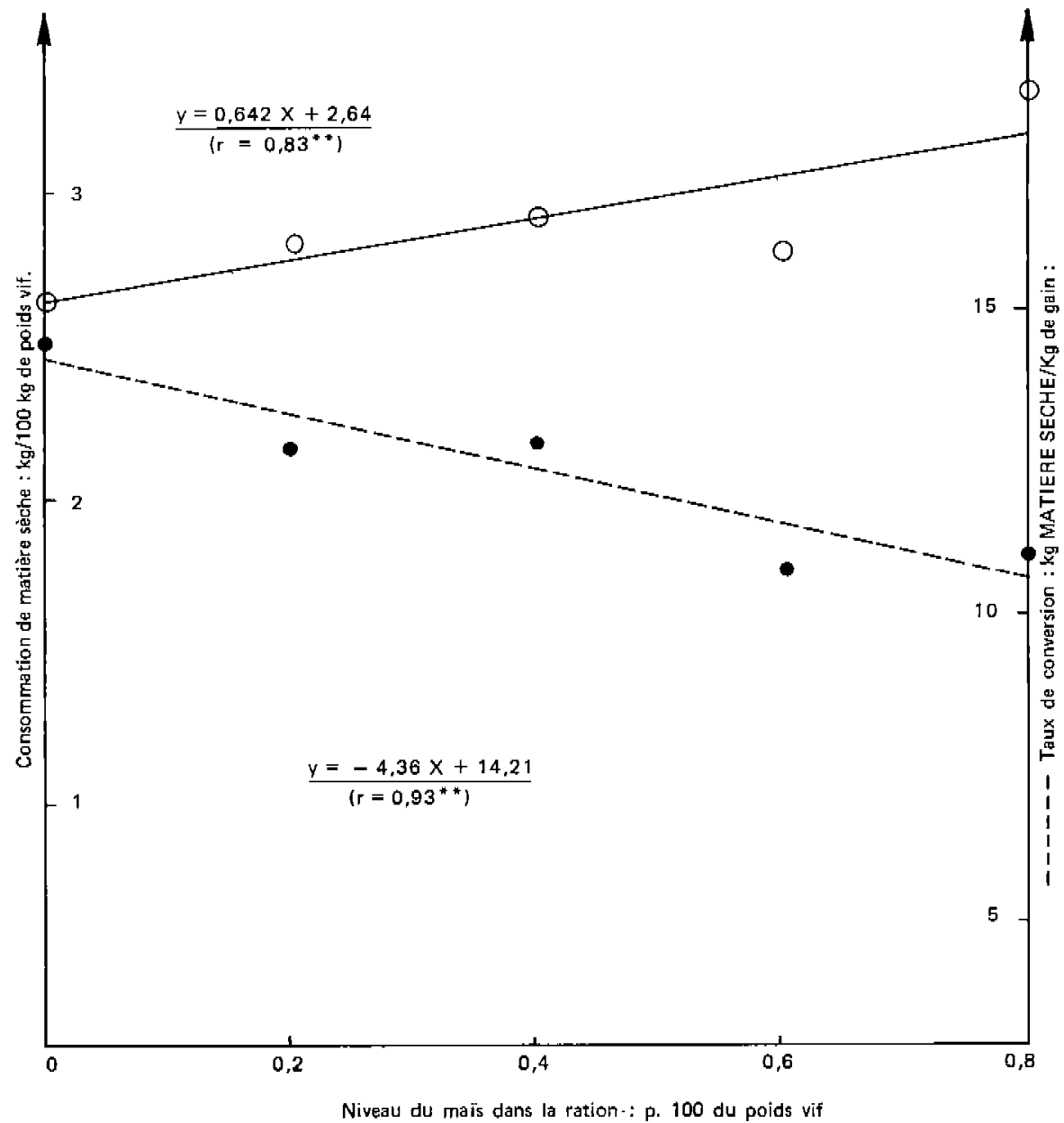

Les résultats techniques ont été nettement améliorés comme l'indiquent les graphiques $\pi^{\circ} 3$ et 4 . Malheureusement, sur le plan économique, cette addition de maïs-grain ne s'est pas révélée rentable.

\section{Pratique de l'engraissement à base de mélasse}

Compte tenu de tous les facteurs étudiés précédemment, le plan d'alimentation que l'on peut actuel lement recommander pour l'engraissement des bovins à base de mélasse est le suivant :

Plan d'alimentation de taurillons engraisșés à base de mélasse liquide.

\begin{tabular}{|c|c|c|c|c|}
\hline Sematne & $\begin{array}{c}\text { Fourrage frais } \\
\text { [kg }]\end{array}$ & $\begin{array}{l}\text { MẽIasse-Urée } \\
{[\mathrm{kE}] \quad(1)}\end{array}$ & $\begin{array}{l}\text { Supplément } \\
\text { protidique (g) }\end{array}$ & $\begin{array}{l}\text { Mélange } \\
\text { míneral (2) }\end{array}$ \\
\hline 1ère et 2 e semaine & ad zibitum & $a d$ izbitum & $500 \mathrm{~g}$ & $a d$ tibitum \\
\hline 3e semaine & $25 \mathrm{~kg}$ & $"$ & $"$ & $"$ \\
\hline 4日 semaine & $\begin{array}{l}2 \text { p.100 } \\
\text { Podds vif }\end{array}$ & $"$ & $"$ & " \\
\hline Se a 8 e semaine & $"$ & $"$ & $400 \mathrm{~g}$ & $"$ \\
\hline Ge semaine Jusqu'à I'abattage & $"$ & $"$ & 300 g & $"$ \\
\hline
\end{tabular}

[1] Urée 2 p.100 à 2,5 p.100 de la mélasse

(2) Phosphate bicalcique : 500

SeI $\ldots \ldots \ldots \ldots \ldots$ : 400

Zn $\mathrm{CD}_{3} \ldots \ldots \ldots \ldots$ : 20

$\mathrm{Fe} \mathrm{SD}_{4}-5 . \mathrm{H.O} \quad \ldots: 27$

$\mathrm{Mn} \mathrm{SO}_{4}-\mathrm{H}, \mathrm{O} \quad \ldots: \mathrm{E}: 23$

Cu $\mathrm{SO}_{4}-5 . \mathrm{H} . \mathrm{O} \quad \ldots:$ : 10

simplement

ph. bicalcique : 500

$\mathrm{Co} \mathrm{SO}_{4}-7 . \mathrm{H.O} \quad \ldots$ : : 0.1

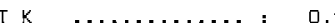

Farine de mañs $\ldots .$. : 19.8 


\section{CONCLUSION}

On peut dire que le système d'engraissement des bovins à base de mélasse liquide est actuellement opérationnel. Il exige certes une certaine technicité de la part de l'éleveur, mais il peut représenter un système de production intéressant pour de nombreux pays sucriers.

Enfin, en terminant cet exposé, il est intéressant de mentionner de nouvelles perspectives qui se dessinent actuellement en ce qui concerne :

- l'utilisation de la bagasse comme fourrage après amélioration de sa digestibilité par des traitements:

- à la soude (Porto-Rico)

- à la vapeur (île Maurice).

Nous avons pu entretenir des génisses pendant 4 mois avec une ration comprenant :

- 85 p. 100 de bagasse traitée à la vapeur.

- 12 p. 100 de mélasse,

- 3 p. 100 d'urée.

- l'utilisation de la canne à sucre elle-même après séparation de la moelle et de la fibre (îles Barbades), la moelle seule étant distribuée aux animaux, la fibre pouvant être récupérée pour la fabrication de panneaux de qualité.

\section{BIBLIOGRAPHIE}

1. Commonwealth Agricultural Bureaux, 1968, « Nutritive value of sugar-cane by-products for ruminants $»$. Annotated bibliography $\mathrm{N}^{\circ} 3$. Commonwealth Bureau of Animal Nutrition, Aberdeen (England).

2. FINCHAM (J.-E.), "Notes on the nutritionnal value of Rhodesian molasses ", Rhodesian Asticultural Journal, Vol. 68, Nu 5, p. 106.
3. MARTIN, PRESTON et WILLIS, 1968, Intensive beef production from sugar-cane : 6 Napier or maize as forage sources at two levels in diet based on molassesurea. Revista Cubana de Ciencia Agricole (English Edition), Vol. 2, $\mathrm{N}^{\circ} 2$, pp. 175-181.

4. Maurilius Sugar Industry Research Institute, 1961. Technical Circular $\mathrm{N}^{\circ}$ 18, December. "By-products of the sugar industry in Mauritius ", p. 147

5. MORRISON (S.-H.), 1967, 1967-1968 ingredient analysis and estimated feed value table for beef, sheep rations, Feedstuffs, 25, November, p. 39 (cité par Preston, 1972).

6. National Research Council (1956). Publication $\mathrm{N}^{\circ} 449$, cité par Fincham (2).

7. PICCIONNI M., 1965, Dictionnaire des aliments pour les animaux, Ed. Agricole, Bologna, traduction française par $J$. Hardouin, pp. 360-366.

8. PRESTON (T.-R.), 1972, "L'engraissement des bovins de boucherie à la mélasse dans les régions tropicales, Revue Mondiale de Zootechnie, F.A.O. No 1, pp. 24-29.

9. PRESTON, ELIAS and WILLIS, 1968, «Intensive beef production from sugar-cane : 7 - The performances of bulls given high levels of molasses-urea at different dilutions ". Revista Cubana de Ciencia Agricola, Vol. 2, No3, pp. 263-268.

10. PRESTON and MOLINA, 1972, "Repeseed meal in molasses-urea based diets for fattening cattle " cité par Preston in "Molasses as the major energy source for beef cattlc ", F.A.O., 1972.

11. PRESTON and WILIS, 1969, "Sugar cane as an energy source for the producton of meat ", An outlook on Agriculture, 6, pp. 29-35.

12. PRESTON and WILLIS, 1970, "Intensive beef production ", Pergamon Press Ltd, 544 pages.

13. REDFERN D., 1972, Projet F.A.O., Nakuru, Kenya Communication personnelle)

13. REDFERN (D.), 1972, Projet F.A.O., Nakurt, Kenya comme aliment des bovins ", Revue agricole et sucrière de l'île Maurice, Vol. 52, No 1 , pp. 15-38.

Les références marquées du signe * dans le texte concernent des domnées non encore publiées. 